\title{
COMPACT WEIGHTED COMPOSITION OPERATORS ON BANACH LATTICES
}

\author{
WILLIAM FELDMAN
}

(Communicated by John B. Conway)

\begin{abstract}
A characterization of compact (and $M$-weakly compact) weighted composition operators on real and complex Banach lattices which can be appropriately realized as function spaces is provided.
\end{abstract}

The compact weighted composition operators on $C^{b}(X)$, all bounded continuous complex valued functions on $X$ with the supremum norm, have been characterized in the case of compact $X$ by Kamowitz [4], and for completely regular $X$ by Singh and Summers [6]. We recall that an operator $T$ on $C^{b}(X)$ is called a weighted composition operator if there exists an $r$ on $C^{b}(X)$ and a continuous map $\phi$ from $X$ to $X$ so that for each $f$ in $C^{b}(X)$, we have $T f(x)=r(x) f(\phi(x))$.

In this note we will characterize a class of compact weighted composition operators (and compact composition operators) on more general function spaces, which we will call Banach $F$-lattices. These include the $L^{p}$-spaces and the Banach lattices, both real and complex, with quasi-interior points (more generally, topological order partitions). We also demonstrate that these operators are equivalent to the $M$-weakly compact weighted composition operators.

We will call a complex Banach space $E$ a Banach $F$-lattice if there exists a real Banach lattice $G$, which can be identified with equivalence classes of real valued functions on a completely regular space $X$, and $E=G+i G$ can be identified with equivalence classes of complex valued functions on $X$ satisfying the following conditions:

(i) If $f$ is equivalent to $g$, then $\{x \in X: f(x)=g(x)\}$ is dense.

(ii) Each continuous complex valued bounded function on $X$ (or function with compact support in the case of a locally compact $X$ ) represents an element in $E$.

(iii) The vector space operations on $E$ and the lattice operations on $G$ correspond to the pointwise defined operations.

Received by the editors September 26, 1988. Presented to the American Mathematical Society at the 94th Annual meeting on January 8, 1988.

1980 Mathematics Subject Classification (1985 Revision). Primary 47B05, 47B38, 46B30. 
(iv) For each $f$ in $E$, the function $|f|(x)=|f(x)|$ is a well-defined element in $E$ and $\|f\|=\||f|\|$.

(v) Given $h \geq 0$, a bounded continuous real valued function on $X$ and $f$ in $E$, the pointwise product $h f$ is a well-defined element of $E$.

The $L^{p}$ spaces are examples of Banach $F$-lattices. We will verify that Banach lattices (complex as well as real) with locally compact representation spaces are also Banach $F$-lattices.

We recall (see [2] or [5]) that a real Banach lattice $G$ is said to have a locally compact representation space $X$ if the space $C_{k}(X, \mathbf{R})$, all real-valued continuous functions on $X$ with compact support, can be identified with a dense (order) ideal in $G$. In fact, $G$ has a locally compact representation space if and only if $E$ contains a topological order partition (see [2]). We will say that a complex Banach lattice (in the sense of Schaefer [5]) has a locally compact representation space $X$ if $E=G+i G$ and $G$ has a locally compact representation space $X$.

Given that $E$ has a locally compact representation space $X$, the real Banach lattice $G$ can be identified with continuous extended real-valued functions on $X$ (i.e. range the two point compactification of $\mathbf{R}$ ), each finite on a dense subset. Thus $E$ can be identified with continuous functions from $X$ to the one point compactification of $\mathbf{C}$, each finite on a dense subset of $X$. Now for $f$ in $E$, a complex valued function $g$ will be said to be equivalent to $f$ if $\{x \in X: f(x)=g(x)\}$ is a dense open subset of $X$. We will now establish that $E$ is a Banach $F$-lattice via the identification of each $f$ in $E$ with this equivalence class of complex functions on $X$.

Lemma. Let $E$ be a complex Banach lattice with locally compact representation space $X$. Then $E$ is a Banach F-lattice.

Proof. We note that condition (iv) follows from the definition of a complex Banach lattice (see [5, p. 137]) and thus we need only verify that (v) is satisfied. Let $h \geq 0$ be a bounded continuous real-valued function on $X$ and let $f$ be in $E$. Then $f=f_{1}+i f_{2}$ as a function on $X$ where $f_{1}$ and $f_{2}$ are real-valued. We view $f_{1}$ as a continuous function from $X$ to the two point compactification of $\mathbf{R}$ and choose an increasing net $\left(f_{\alpha}\right)$ of functions in $C_{k}(X, \mathbf{R})$ converging up to $f_{1}$ in norm and pointwise for all $x$. Now $\left(h f_{\alpha}\right)$ is a Cauchy net since $\left|h f_{\alpha}-h f_{\beta}\right| \leq\|h\|_{\infty}\left|f_{\alpha}-f_{\beta}\right|$ so that $\left\|h f_{\alpha}-h f_{\beta}\right\| \leq\|h\|_{\infty}\left\|f_{\alpha}-f_{\beta}\right\|$. Hence $\left(h f_{\alpha x}\right)$ converges to some element $g$ in $G$ which we identify with an extended real valued function. If there exists an $x_{0}$ in $X$ such that $g\left(x_{0}\right)>h f_{1}\left(x_{0}\right)$ then $g$ exceeds $h f_{1}$ on a compact neighborhood $N$ of $x_{0}$. Let $k \geq 0$ be in $C_{k}(X, \mathbf{R})$ such that $k$ vanishes off of $N$, and $\left(g-h f_{1}(x)\right) \geq k(x)$ for all $x$. Now $\left(g-h f_{\alpha}\right)$ exceeds $k$ for all $x$ and $\alpha$, so that $\left\|g-h f_{\alpha}\right\| \geq\|k\|$, a contradiction. Hence $g \leq h f_{1}$. If there exists an $x_{0}$ such that $g\left(x_{0}\right)<h f_{1}\left(x_{0}\right)$, then $g\left(x_{0}\right)<h f_{\hat{\hat{\alpha}}}\left(x_{0}\right)$ for some $\hat{\alpha}$. Again $g<h f_{\hat{\alpha}}$ on a neighborhood $N$ of $x_{0}$, and we choose a function $k \geq 0$ with $k<\left(h f_{\hat{\alpha}}-g\right)$. Thus $\|k\| \leq\left\|h f_{\alpha}-g\right\|$ 
for all $\alpha>\hat{\alpha}$, and we conclude that $h f_{1}=g$. A similar argument applies to $f_{2}$.

Given $E$ and $F$ Banach $F$-lattices identified with equivalence classes of complex valued functions on $X$ and $Y$ respectively, we will call a bounded operator $\Phi$ from $E$ to $F$ a composition operator if there exists a continuous function $\phi$ from $Y$ to $X$ so that

$$
\Phi(f)(y)=f(\phi(y))
$$

for each $f$ in $E$ and $y$ in $Y$. A bounded operator $T$ will be called a weighted composition operator if there exists a continuous complex valued function $r$ on $Y$ and a composition operator $\Phi$ that

$$
T(f)(y)=r(y) \Phi f(y)=r(y) f(\phi(y))
$$

We will often write $T=r(f \circ \phi)$.

A weighted composition operator $T$ will be said to satisfy condition $(*)$ if there exists a $\delta \geq 0$ so that for each $x$ in $\phi(Y)$ and neighborhood $N$ of $x$ there is an element $f$ in $E$ with $\|f\|=1$ equivalent to a function vanishing outside of $N$, and $\|\Phi(f)\| \geq \delta$.

Although many operators will satisfy condition $(*)$, for example, translations or shifts, we cite an elementary example where this condition fails. On $L^{1}[0,1]$, let $\Phi(f)=f(\sqrt{x})(\phi(x)=\sqrt{x})$. At $x=0$ condition $(*)$ is not satisfied.

Theorem 1. Let $E$ and $F$ be Banach $F$-lattices identified with equivalence classes of functions on $X$ and $Y$ respectively, and $T$ a weighted composition operator from $E$ to $F$. Let $r$ be in $C(Y)$, and $\phi$ a continuous map from $Y$ to $X$, so that $T f=r(f \circ \phi)$ for each $f$ in $E$. If $T$ satisfies condition $(*)$ and for each $\varepsilon>0$, there is a positive $\hat{\varepsilon}$ less than $\varepsilon$, so that $\overline{\phi\{y:|r(y)| \leq \hat{\varepsilon}\}}$ is disjoint from $\phi\{y:|r(y)| \geq \varepsilon\}$, then the following statements are equivalent:

(i) $T$ is a compact operator.

(ii) For each $\varepsilon>0$, the set $\phi\{y:|r(y)|>\varepsilon\}$ is finite.

Proof. We first assume that condition (ii) is not satisfied. Thus there exists an $\varepsilon>0$ and a sequence $\left(a_{i}\right)$ of distinct points in the image of $\{y:|r(y)| \geq \varepsilon\}$ under the map $\phi$. We construct a collection of disjoint closed neighborhoods for an infinite collection of points in $\left\{a_{i}\right\}$ as follows. Choosing a subsequence if necessary, we assume that no subsequence of $\left(a_{i}\right)$ converges to a point in $\left\{a_{i}\right\}$. Now let $N_{1}$ be a neighborhood of $a_{1}$ such that $\left\{a_{i}\right\}-N_{1}$ is an infinite set and $N_{1}$ is disjoint from the set $F=\overline{\phi\{y:|r(y)|<\hat{\varepsilon}\}}$. Let $a_{i_{2}}$ be a point in $\left\{a_{i}\right\}-N_{1}$ and choose a closed neighborhood $N_{2}$ of $a_{i_{2}}$ disjoint from $N_{1}$ and $F$ such that $\left\{a_{i}\right\}-\left(N_{1} \cup N_{2}\right)$ is an infinite set. Continuing inductively, let $N_{j}$ be a neighborhood of a point $a_{i_{j}}$ where $a_{i_{j}}$ is in the infinite set $\left\{a_{i}\right\}-$ $\bigcup_{k<j} N_{k}$, disjoint from $\bigcup_{k<j} N_{k}$ and $F$ such that $\left\{a_{i}\right\}-\bigcup_{k \leq j} N_{k}$ is infinite. Now condition (*) implies that for each $i$ there exists an $f_{i}$ in $E$ with $f_{i}$ 
equal to zero on the complement of $N_{i},\left\|f_{i}\right\|=1$, and $\left\|\Phi\left(f_{i}\right)\right\| \geq \delta$. Now for $j \neq k$,

$$
\left\|T f_{j}-T f_{k}\right\|=\left\|r\left(f_{j} \circ \phi\right)-r\left(f_{k} \circ \phi\right)\right\| \geq\left\|r\left(f_{j} \circ \phi\right)\right\| \geq \hat{\varepsilon}\left\|\Phi\left(f_{j}\right)\right\| \geq \hat{\varepsilon} \delta
$$

since $f_{j}$ and $f_{k}$ have disjoint support and $|r|$ exceeds $\hat{\varepsilon}$ on the points where $\left(f_{j} \circ \phi\right)$ is nonzero. Thus the sequence $\left(T f_{i}\right)$ has no convergent subsequence.

Assume that (ii) is satisfied. Given $\varepsilon>0$, let $H$ be a positive continuous real-valued function on $Y$ which is one on $\{y:|r(y)| \geq \varepsilon\}$ and zero on $\{y:|r(y)|<\varepsilon / 2\}$, and bounded by one, (e.g., $\left.2 / \varepsilon\left(|r|-\frac{1}{2} \varepsilon\right) \wedge \frac{1}{2} \varepsilon\right)^{+}$. Now for $f$ in the unit ball of $E$

$$
\|H T f-T f\| \leq\|\varepsilon|f \circ \phi|\| \leq \varepsilon\|\Phi\|,
$$

where $\|\Phi\|$ is the operator norm of $\Phi$. We will complete the proof by showing that $H T$ is a finite rank operator (i.e., $T$ is the limit of finite rank operators). Let $\phi\{y:|r(y)| \geq \varepsilon / 2\}$ be denoted by $\left\{a_{i}: i=1,2, \ldots, n\right\}$ and for each $i$ choose a continuous bounded real-valued function $L_{i}$ so that $L_{i}\left(a_{i}\right)=1$ and $L_{i}\left(a_{j}\right)=0$ for $i \neq j$. Now for each $f$ and $i$, there exists a constant $\alpha_{i}(f)$ so that

$$
H(y) r(y)\left(L_{i} \circ \phi\right)(y)(f \circ \phi)(y)=\alpha_{i}(f) H(y) r(y)\left(L_{i} \circ \phi\right)(y)
$$

for all $y$ in $Y$. Hence $H T f=\sum_{i=1}^{n} \alpha_{i}(f) H r\left(L_{i} \circ \phi\right)$.

Corollary. Let $E$ and $F$ be Banach $F$-lattices of functions on $X$ and $Y$ respectively. Let $\Phi$ be a composition operator such that $\Phi(f)=f \circ \phi$ for $\phi$ a continuous map from $Y$ to $X$ and $f$ in $E$. If $\Phi$ satisfies condition $(*)$, then $\Phi$ is compact if and only if the image of $Y$ under $\phi$ is finite.

In the special case that $E$ and $F$ are spaces of all continuous bounded functions on a completely regular space with the sup-norm topology, the $(*)$ assumption and the separation assumption are not necessary in the previous results. Thus we provide an alternative proof for the result established by Singh and Summers in [6], and, in a slightly different form, by Kamowitz for compact $X$ in [4].

Theorem 2. Let $C^{b}(X)$ and $C^{b}(Y)$ be the spaces of all continuous complex valued bounded functions on $X$ and $Y$ respectively with the sup-norm topology. Let $\phi$ be a continuous map from $Y$ to $X$ and $r$ a continuous bounded complex valued function on $Y$.

(a) The composition operator $\Phi$ defined by $\Phi(f)=f \circ \phi$ for each $f$ in $C^{b}(X)$ is compact if and only if the image of $Y$ under $\phi$ is finite.

(b) The weighted composition operator $T$ defined by $T f=r(f \circ \phi)$ for each $f$ in $C^{b}(X)$ is compact if and only if for each $\varepsilon>0$, the image under $\phi$ of $\{y:|r(y)| \geq \varepsilon\}$ is finite.

Proof. We need only prove part (b) and proceed as in the proof of Theorem 1. Assume first that there is an infinite sequence of distinct points $\left(a_{i}\right)$ in the 
image of $\{y:|r(y)| \geq \varepsilon\}$ under the map $\phi$. We choose neighborhoods $N_{i}$ as in the proof of Theorem 1 without regard to the set $F$ (i.e., without $N_{i}$ disjoint from $F$ ). Then the function $f_{j}$ is chosen to be one at $a_{i_{j}}$ and zero on the complement of $N_{i}$ with $0 \leq f_{j} \leq 1$. For $y$ in $Y$ with $r(y) \geq \varepsilon$ and $\phi(y)=a_{i_{j}}$ we have

$$
\left\|T f_{j}-T f_{k}\right\| \geq\left\|r\left(f_{j} \circ \phi\right)-r\left(f_{k} \circ \phi\right)\right\| \geq\left|r(y) f_{j}\left(a_{i_{j}}\right)\right|=|r(y)| \geq \varepsilon .
$$

The proof of the converse is the same as the proof in Theorem 1.

We recall that (e.g., see [1, p. 313]) that an operator from $E$ to $F$ is called $M$-weakly compact if for every disjoint sequence $\left(f_{n}\right)$ in $E$ bounded in norm, the sequence $\left(\left\|T\left(f_{n}\right)\right\|\right)$ converges to zero ( $f$ is disjoint from $g$ if $|f| \wedge|g|=0$ ). We can now prove the following:

Theorem 3. A weighted composition operator $T$ satisfying the hypothesis of Theorem 1 is compact if and only if $T$ is $M$-weakly compact.

Proof. We will verify that $T$ is $M$-weakly compact if and only if condition (ii) of Theorem 1 is satisfied. Given that (ii) is not satisfied, the same argument as in the proof of Theorem 1 yields a bounded disjoint sequence whose image under $T$ does not converge to zero in norm. Given that (ii) is satisfied, let $H$ be the function in the proof of Theorem 1. We note that for $\left(f_{i}\right)$ a disjoint sequence bounded in $E$, the sequence $\left(H T f_{i}\right)$ is also disjoint. Since $\phi\{y: r(y) \geq \varepsilon / 2\}$ is finite, at most finitely many of the functions $H T f_{i}$ can be nonzero. Thus $\left(H T f_{i}\right)$ is convergent to zero in norm. Now $H T$ is $M$-weakly compact and since the $M$-weakly compact operators are closed (e.g., [1, p. 317]), we conclude that $T$ is $M$-weakly compact.

\section{REFERENCES}

1. C. D. Alliprantis and O. Burkinshaw, Positive operators, Academic Press, Orlando, 1985.

2. W. Feldman and J. F. Porter, Banach lattices with locally compact representation spaces, Math. Z. 174 (1980), 233-239.

3. __ Operators on Banach lattices as weighted composition operators, J. London Math. Soc. 33 (1986), 149-156.

4. H. Kamowitz, Compact weighted endomorphisms of $C(X)$, Proc. Amer. Math. Soc. 83 (1981), 517-521.

5. H. H. Schaefer, Banach lattices and positive operators, Springer-Verlag. New York. 1974.

6. R. K. Singh and W. H. Summers, Compact and weakly compact composition operators on spaces of vector valued continuous functions, Proc. Amer. Math. Soc. 99 (1987), 667-670.

Department of Mathematical Sciences, University of Arkansas, Fayetteville, ARKANSAS 72701 